\title{
A06
}

\section{Integrated Top Seal Evaluation for Robust} Solutions

\author{
J.S. Davis* (ExxonMobil Upstream Research Co.), S.A. Barboza \\ (ExxonMobil Upstream Research Co.) \& X. Liu (ExxonMobil Upstream \\ Research Co.)
}

\section{SUMMARY}

Prediction of hydrocarbon column heights and contacts requires simultaneous evaluation of capillary and mechanical bed seal properties together with trap geometry (crest, spill), estimation and quantification of uncertainties, and propagation of these uncertainties through the analysis. We developed a method for making a probabilistic determination of total seal capacity for a hydrocarbon trap, simultaneously considering both bed seal leak mechanisms (capillary entry pressure and hydraulic fracture pressure), and accounting for trap geometry. Assuming unlimited gas and oil charge and uniform seal properties, simultaneous evaluation of top seal capacity and geometric controls on hydrocarbon fill, leads to recognition of only six possible combinations of hydrocarbon fill control in two-phase (gas and oil) systems. Single phase systems are controlled by only one of the three leak mechanisms.

Different combinations of top seal and geometric spill controls can achieve very similar final hydrocarbon distributions in hydrocarbon traps. Without investigating all possible controls it is easy to draw incorrect conclusions about controls on hydrocarbon fill in any single accumulation, promoting incorrect prognostications about undrilled traps. When considering field development plans that include pressure support, misunderstanding actual controls on fluid distributions could lead to unanticipated outcomes, such as leakage through the top seal. 
Understanding and predicting total hydrocarbon column height and contacts in a hydrocarbon trap occupies the attention of every hydrocarbon exploration company. We now recognize that such predictions require simultaneous evaluation of capillary and mechanical bed seal properties together with fault seal evaluation, careful estimation and quantification of uncertainties, and propagation of these uncertainties through the analysis. We have developed, and patented, a method (Figure 1) for making a probabilistic determination of total seal capacity for a hydrocarbon trap, simultaneously considering both bed seal leak mechanisms (capillary entry pressure and hydraulic fracture pressure), and accounting for trap geometry.

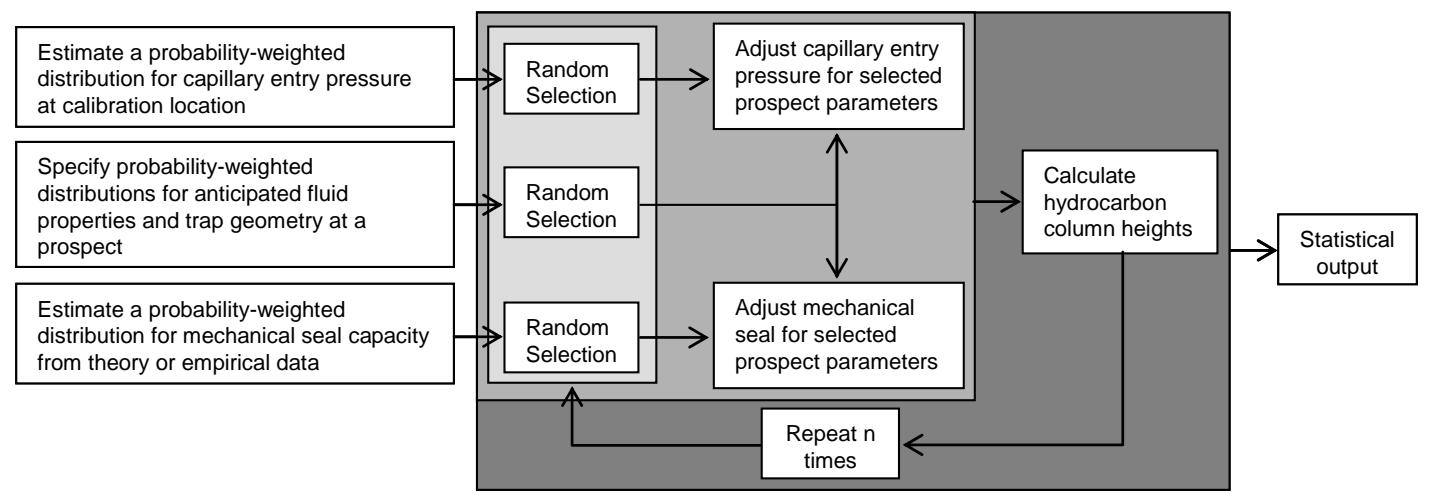

Figure 1 Simplified model for simultaneous probabilistic evaluation of capillary and mechanical seal capacity and trap geometric controls on hydrocarbon fill.

Hydrocarbon contact predictions are sensitive to trap geometry (including structural and stratigraphic sand connections) and top seal leak potential. The first step in top seal analysis is to define trap parameters such as crest and synclinal spill depths and to identify potential structural and stratigraphic connection depths. The fracture pressure of the top seal can be established via geomechanical methods or by proxy data such as leak-off tests. In-situ hydrocarbon pressure gradients are established either by regression of measured subsurface fluid pressures or by estimation from PVT analyses. PVT data, in combination with mercury porosimetry, compositional data, or by inference from known fluid contacts, may be used to estimate top seal capillary entry pressure.

With simplifying assumptions of no gas or oil charge limitation and uniform seal properties, the aforementioned parameters become the primary control on hydrocarbon fill. Several deductions follow. First, we recognize only six possible combinations of hydrocarbon fill control in two-phase (gas and oil) systems (Figure 2). Second, we find that the order of gas and oil fill does not impact the final fluid distribution. Finally, fluid distributions in single phase systems are a trivial reduction of the multi-phase problem.

Figures 2A, 2B, and 2C depict potential outcomes in which structural relief exceeds both the mechanical and capillary seal capacity with respect to either hydrocarbon phase. Figure 2A depicts a case in which the HFP (hydraulic fracture pressure) < OEP (oil entry pressure) $<$ GEP (gas entry pressure). In this case oil fills the trap to a column height whose absolute pressure exceeds the HFP at the crest of the trap. Additional charge of either oil or gas will leak from the trap. Figure 2B depicts a case in which OEP < GEP < HFP (Sales (1997) Class 3 traps). The gas and oil columns of traps in this category are limited by the capillary entry pressure at the crest and the gas-oil contact, respectively. Figure 2C depicts a case in which the OEP $<$ HFP $<$ GEP. This class of traps has gas columns limited by HFP at the crest and oil columns limited by OEP at the gas-oil contact.

Figures 2D, 2E, and 2F depict potential outcomes in which the hydrocarbon column is limited by the structural spill point. Figure 2D depicts a case in which OEP $<$ GEP $<$ HFP (Sales (1997) Class 2 traps). In this case the buoyancy of the gas column exceeds the GEP at the crest and the buoyancy of the oil column is less than the OEP at the gas-oil contact. Gas leaks at the crest of the trap and oil is filled to spill. Figure 2E depicts a case in which OEP $<$ HFP $<$ GEP. The absolute pressure of the gas column for traps in this category exceeds the 

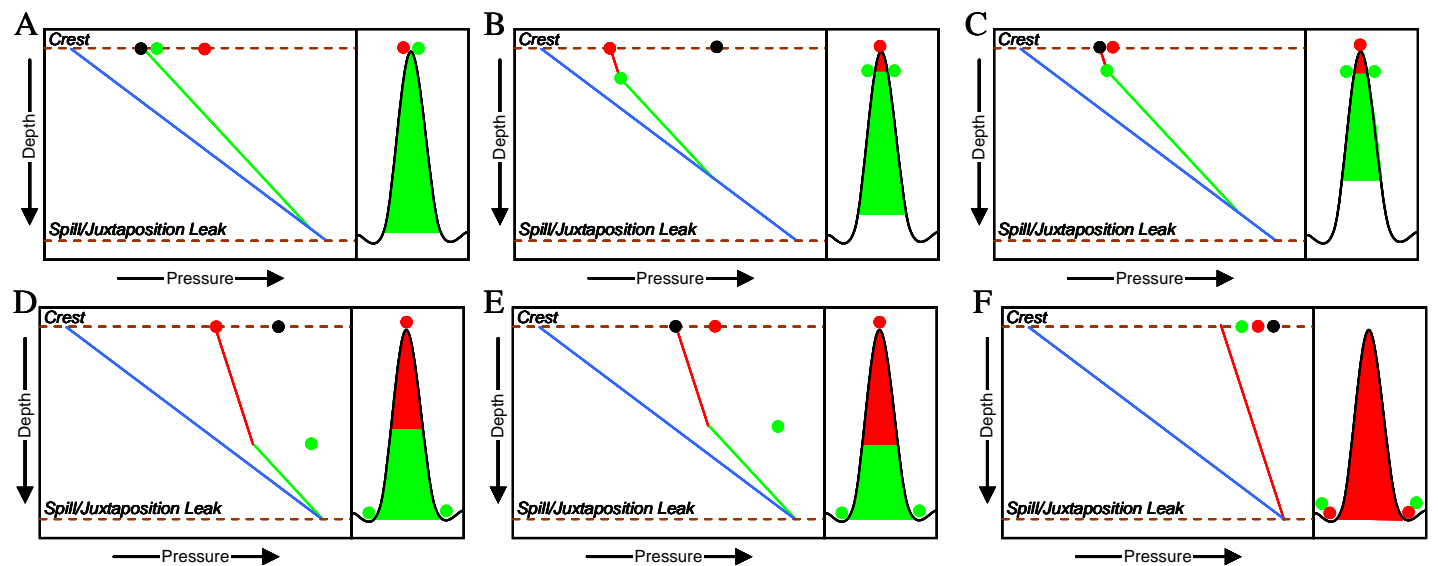

Figure 2 Six possible multi-phase (gas and oil) fill scenarios with leak mechanisms that control accumulation of each phase. Scenarios B, D, and F follow Sales (1997). Red, green, and blue lines are gas, oil, and water gradients, respectively. Figure to the right of the depthpressure plot is a simple trap cross section (crest and spill depths correspond to the depthpressure plot. Trap fills are red for gas, green for oil. Red and green dots on depth pressure plots correspond to the gas and oil entry pressures, respectively. The black dot is hydraulic fracture pressure for the phase whose gradient intersects it. Red and green dots on the trap cross-section represent location gas and oil leaks, respectively.

HFP at the trap crest and oil is filled to spill. Figure 2F depicts a case in which the buoyancy of the gas column is less than both the GEP and the HFP (Sales (1997) Class 1 traps). This class of traps is filled to spill with gas.

The relative timing of gas or oil charge does not affect the final arrangement of fluid contacts. Consider the case depicted in Figure 2E, for example. A primary oil charge would first fill the trap with oil to the structural spill point. A subsequent gas charge would displace the oil downward and out of the trap. The gas column would grow until the buoyancy pressure of the gas column was limited by the HFP at the crest, yielding the final equilibrium configuration depicted in Figure 2E. If the relative timing were reversed, the trap would first be filled with a gas column limited by the HFP at the crest. The buoyancy exerted by a subsequent oil charge would displace gas through the crest of the structure and the oil column would grow until it reached the structural spill point, again yielding the final equilibrium configuration depicted in Figure 2E.

It is clear that trap architectural elements, rock mechanical strength, and top seal capillary entry pressure may combine in various ways to produce very similar equilibrium hydrocarbon distributions in traps. A clear understanding of the possible geologic scenarios that may have led to the preproduction distribution of in-place hydrocarbons demands a careful investigation of the contributing parameters as well as their uncertainties. Without this analysis it is very easy to draw the incorrect conclusions about the control on hydrocarbon contacts in any single accumulation, promoting incorrect forecasts for undrilled traps.

When field development plans include gas or water injection for pressure maintenance, misunderstanding the fundamental controls on fluid column heights could lead to unintended outcomes. For example, failure to recognize a gas cap limited by HFP (e.g., Fig. 2C) may lead to losses of injected gas through fractures in the top seal. An early understanding that the HFP impinges on in-place hydrocarbon columns could influence the field development plan by modifying the pressure maintenance strategy to mitigate injected gas loss.

Reference: Sales, J.K. [1997] Seal strength vs. trap closure - a fundamental control on the distribution of oil and gas. In: R.C. Surdam (Ed.) Seals, Traps, and the Petroleum System, AAPG Memoir 67, 57-83. 